\title{
Investigation of the Factors Affecting Quality of Life in Patients with Mild to Moderate Alzheimer's Disease: A Cross-Sectional Study from Turkey
}

Elvan Felekoğlu ( $\square$ elvanfelekoglu@gmail.com )

Dokuz Eylul University, Institute of Health Sciences, Physical Therapy and Rehabilitation https://orcid.org/0000-0001-6633-1572

Sevgi ÖZALEVLİ

Dokuz Eylul University, School of Physical Therapy and Rehabilitation

Hazal YAKUT

Dokuz Eylul University, School of Physical Therapy and Rehabilitation

RIdvan AKTAN

Izmir University of Economics, Department of Physiotherapy

Görsev YENER

Izmir University of Economics, Faculty of Medicine

Research

Keywords: Alzheimer's Disease, Health Related Quality of life, Caregiver

Posted Date: May 11th, 2021

DOI: https://doi.org/10.21203/rs.3.rs-486899/v1

License: (c) (i) This work is licensed under a Creative Commons Attribution 4.0 International License.

Read Full License 


\section{Abstract \\ Background}

Like other chronic diseases with limited medical treatment, the most important goal of Alzheimer's disease rehabilitation is to provide a better quality of life. The purpose of this study was to investigate the factors affecting the quality of life of the patients with Alzheimer's Disease (according to parts of the Quality of Life in Alzheimer' Disease Questionnaire: patient, proxy and total score).

\section{Methods}

In this cross-sectional study, 73 home-dwelling patients with Alzheimer's Disease and their caregivers were recruited. Cognition, depression and Quality of Life in Alzheimer' Disease -self rating were asked to the patient. The caregiver was asked about patient's sociodemographic information, sleepiness, activities of daily living and Quality of Life in Alzheimer' Disease -proxy rating.

\section{Results}

The Quality of Life in Alzheimer' Disease -self rating were higher than the Quality of Life in Alzheimer' Disease -proxy rating. Cognition $(p=0.02)$, sleepiness $(p<0.01)$ and depression $(p=0.03)$ were correlated with the Quality of Life in Alzheimer' Disease -self rating, while the independence level of the patient in activities of daily living was correlated to the Quality of Life in Alzheimer' Disease -proxy rating $(p<0.05)$. Predicting quality of life according to linear regression analysis those were statistically significant in respectively; depression is for Quality of Life in Alzheimer' Disease, depression and cognition were for Quality of Life in Alzheimer' Disease -self rating and instrumental activities of daily living was for QoL-ADPR $(p<0.01)$.

\section{Conclusion}

While subjective situations such as psychology is important for the patients with Alzheimer's Disease, objective conditions such as the independence of the patient's in daily life activities are important for the caregiver. While evaluating the quality of life, both the evaluations of the patients with Alzheimer's Disease and the caregiver should be applied, because different things are prioritized by them and priorities of both should be taken into account when planning the treatment program.

\section{Background}

The incidence of dementia has increased with the aging of the world population (1). Due to dementia, progressive damage occurs in patients' memory, thinking and behaviour, executive functions and daily 
life activities (2). Because of these problems, dementia patients become dependent on someone else, mostly a relative, and need help in order to perform daily life activities (3).

Dementia is one of the most important diseases of our age, which is a burden on the health systems of countries as well as patients and their families (1). Alzheimer's Disease (AD), which is $50-70 \%$ of all dementia cases, is the most common form of dementia. There is no cure for AD yet and the main goal in its treatment is to ensure the optimization of quality of life (4-6).

According to World Health Organization (WHO), Quality of Life (QoL) is an individual's perception of their position in life in the context of the culture and value systems in which they live and in relation to their goals, expectations, standards and concerns (7). Living better with $A D$, is important for the patient, caregiver and health care professions (8).

Quality of Life is an important assessment due to its multidimensional construct and guides healthcare professionals in patient-centred treatment (9). Due to its multidimensionality and culture specificity, QoL data may vary in different populations. "Adding life to years rather than years to life", as a philosophy of healthy aging, emphasizes the importance of quality of life. It is not possible to reach only a standard result for the QoL (9). It is inevitable to evaluate the QoL and its associated factors in different populations even though the same disease.

Evaluation of QoL of People with AD (PwAD) is a controversial issue, as the QoL is an individuals' perception. While some studies suggest that the quality of life should be evaluated by the PwAD's relative or primary caregiver, some suggest that it is a subjective data and the patient should express himself (6, 8,10 ). Studies show that mild-to-moderate PwAD (with Mini Mental status assessment of 10 points or more) can provide accurate information about quality of life $(11,12)$.

Based on the aforementioned situations, the purpose of this study is to evaluate and interpret the factors affecting the QoL of PWAD in terms of patients and caregivers.

\section{Methods}

\section{Participants}

In this prospective, cross-sectional study, nonprobability convenience sampling was used for sample selection. Seventy-three PWAD who were diagnosed with mild to moderate AD according to The National Institute of Neurological and Communicative Disorders and Stroke and the Alzheimer's Disease and Related Disorders' Association Criteria and their caregivers were recruited in this study.

The answers of the patients whose mini mental state examination (MMSE) scores are 10 and higher are more reliable. Based on this situation, the inclusion criteria of the patients were determined as follows: Getting at least 10 points of MMSE, having mild to moderate AD and stability in clinical situations for psychotropic drug users. The exclusion criteria of the patients were: Getting diagnosed with other types of 
dementia or other neurological diseases, having a comorbid disease (cardiopulmonary disease, orthopaedic disease etc.) that will may create caregiver burden, having behavioural problems (yelling, wandering, being aggressive, etc.), having communication problems (such as seeing, hearing). The inclusion criteria of caregiver were living with patient and the exclusion criteria was having communication problems such as visual, hearing, or speech impairments.

As the sample size was at least 10 times the number of variables, a post-hoc power analysis was performed when 73 cases were reached. The power of the study was evaluated using G. Power 3.1. It was found to be 0.99 for QoL-AD-SR (sample size of 73, effect size of 0.5 , and alpha value of 0.05 were considered) and was found 0.96 for QoL-AD-PR (sample size of 73, effect size of 0.16 , alpha value of 0.05 were considered).

MMSE was applied to the patients during the routine examination. A statement about the study was made to those with mild to moderate stages and their caregivers. The patients' quality of life and depression were evaluated after the consent form was signed by those who agreed to participate. The researchers read the questions for the patients. Sociodemographic information was asked to the caregivers of the patients and written on the data record form. Evaluation forms were given to them for the questions that the caregivers should fill. They were able to ask researchers for information when needed.

\section{Questionnaires}

The sociodemographic information (such as gender, age, disease duration, having another disease) of PwAD was obtained from both of patients and their caregivers. The other assessment parameters were:

Mini Mental State Examination (MMSE):

As a set of 30 questions MMSE, is used to assess cognitive function (indicates orientation, learning, short-term memory, language use, comprehension, and basic motor skills). It ranges from 0 to 30 and low score means high cognitive impairment (13).

Clinical Dementia Rating (CDR) Scale:

This scale is a 5-point scale used to determine the possible stage of dementia: 0 (no dementia), 0.5 (questionable dementia), 1 (mild dementia), 2 (moderate dementia), and 3 (severe dementia). According to assessment of clinician, an overall CDR score may be calculated through the use of an algorithm by the clinician (14).

Quality of Life in Alzheimer's Disease (QoL-AD):

This scale consists of two parts; QoL-AD-SR part filled by patients and QoL-AD-PR part filled by caregivers. It contains 13 domains (physical health, energy, mood, living situation, memory, family, marriage, friends, self as a whole, ability to do chores, ability to do things for fun, money and life as a 
whole) which are rated from poor (1) to excellent (4) and total from 13 to 52 . High score means better QoL. It can be done by mild to moderate PwAD (15).

Activities of Daily Living (ADL):

Barthel ADL scale (ranges from 0 to 100) was used for the skills of the patients to perform basic daily life activities (such as feeding, personal toileting, bathing, dressing and undressing, getting on and off a toilet, controlling bladder, controlling bowel, moving from wheelchair to bed and returning, walking on level surface and ascending and descending stairs) (16). Lawton-Brody Instrumental Activities of Daily Living (IADL) scale (ranges from 0 to 7 ) used for assessing independent living skills (using the telephone, shopping, preparing food, housekeeping, doing laundry, using transportation, handling medications, handling finances) (17). High score means more independence in both tests.

Geriatric Depression Scale (GDS):

Short form of GDS was used to assess the depression of patients. It comprises 15 questions which answered as yes or no. In this scale, 5 questions are positive, while the others are negative. In the evaluation of the scale, no answers to positive questions and yes to negative questions are matched with 1 points. Lower score means better mood (18).

Epworth Sleepiness Scale (ESS):

It is a qualitative and quantitative measurement of sleep to detect excessive daytime sleepiness. Eight questions were scored as $0,1,2,3$ according to the probability of sleeping, and the total was calculated. High score indicates sleepiness. If the total score is greater than 10 , it indicates the presence of pathological sleepiness (19).

\section{Procedures}

This prospective, cross-sectional study was performed in Izmir, a city of west part of Turkey. Answering questionnaires were done at outpatient dementia clinic and it took nearly $30 \mathrm{~min}$.

Approval of the study was obtained from the ethical committee of the Ethics Committee of Dokuz Eylül University. Noninvasive Research Ethics Board (2017/01-27). After participants and their caregivers agreed to participate in the study they signed the written permission.

Caregivers completed EDSS, ADL and QoL questionnaires related to their patients by themselves. PWAD answered the questions about their depression and QoL by verbally.

\section{Statistical Analyses}

All statistical analyses were performed in the Statistical Package for the Social Sciences, version 23.0 for Windows. The Shapiro-Wilk test was used for analysing the normal distribution of variables. If the variables were parametric, they were described by their mean and standard deviation (SD) and if the variables were nonparametric, they were described by their median and interquartile ranges. 
Correlational analyses (Pearson's r) were performed to clarify the relationships between study variables (age, disease duration, cognition, depression, sleepiness, activities of daily living) and QoL.

The stepwise backward multiple linear regression analysis was used to identify independent predictors of QoL. The model fit was assessed using appropriate residual and goodness-of-fit statistics. Statistical significance was assigned at the $p<.05$ level.

\section{Results}

A total of 73 home-dwelling patients and their caregivers were included in the study. In Table 1, the sociodemographic and clinical characteristics of the patients were given.

The 46 (63\%) of 73 patients were female. Forty-six (63\%) of all were mild. The mean age of participants were 68.7 years $(S D=8.53)$ and disease duration was 2.06 years $(S D=1.49)$. The mean MMSE was $19.38 \pm 4.53$. Patients' self-rated quality of life scores were higher than those of their caregivers (Table 1).

Table 1

Sociodemographic and clinical characteristic of participants

\begin{tabular}{|lll|}
\hline Patient characteristic & Mean & SD \\
\hline Gender female (\%) & $46(63)$ & \\
CDR (\%): $1 / 2$ & $46(63) / 27(37)$ & \\
\hline Age (years) & 68.7 & 8.53 \\
\hline Disease duration (years) & 2.06 & 1.49 \\
\hline MMSE & 19.38 & 4.53 \\
\hline GDS & 2.49 & 2.56 \\
\hline ESS & 5.03 & 4.83 \\
\hline ADL & 97.4 & 5.78 \\
\hline IADL & 5.44 & 1.98 \\
\hline QOL-AD-SR & 38.01 & 4.12 \\
\hline QOL-AD-PR & 31.62 & 5.20 \\
\hline QOL-AD & 35.88 & 3.61 \\
\hline
\end{tabular}

SD: Standard Deviation, CDR: Clinical Dementia Rating, MMSE: Mini Mental State Examination, GDS: Geriatric Depression Scale, ESS: Epworth Sleepiness Scale, ADL: Activities of Daily Living, IADL: 
Instrumental Activities of Daily Living, QOL-AD-SR: Quality of Life in Alzheimer's Disease -Self Rated, QOLAD-PR: Quality of Life in Alzheimer's Disease -Proxy Rated QoL-AD: Quality of Life in Alzheimer's Disease. MMSE, GDS, ESS and proxy-rated QoL were correlated with self-rated QoL $(p<0.05)$. ADL, IADL and selfrated QoL were correlated with proxy-rated QoL $(p<0.05)$. There was only GDS correlated with QoL-AD ( $p$ $<0.05$, Table 2).

Table 2

Correlations of QOL-AD, QOL-AD-SR and QOL-AD-PR with study variables

\begin{tabular}{|lllllll|}
\hline Variable & \multicolumn{2}{l}{ QOL-AD-SR } & \multicolumn{2}{l}{ QOL-AD-PR } & QOL-AD \\
\cline { 2 - 8 } & $\mathbf{r}$ & $\mathbf{p}$ & $\mathbf{r}$ & $\mathbf{p}$ & $\mathbf{r}$ & $\mathbf{p}$ \\
\hline Age & -.02 & .432 & .061 & .305 & .014 & .455 \\
\hline Disease duration & -.045 & .351 & .136 & .126 & .031 & .399 \\
\hline MMSE & -.250 & $.017 *$ & .144 & .112 & -.120 & .155 \\
\hline GDS & -.440 & $<.001 *$ & -.101 & .199 & -.383 & $<.001 *$ \\
\hline ESS & -.232 & $.024 *$ & -.017 & .442 & -.184 & .059 \\
\hline ADL & -.185 & .058 & .195 & $.049 *$ & -.047 & .346 \\
\hline IADL & .071 & .276 & .284 & $.007 *$ & .190 & .054 \\
\hline QOL-AD-SR & - & - & .266 & $.011 *$ & .887 & $<.001 *$ \\
\hline QOL-AD-PR & .266 & $.011 *$ & - & - & .682 & $<.001 *$ \\
\hline
\end{tabular}

MMSE: Mini Mental State Examination, GDS: Geriatric Depression Scale, ESS: Epworth Sleepiness Scale, ADL: Activities of Daily Living, IADL: Instrumental Activities of Daily Living, QOL-AD-SR: Quality of Life in Alzheimer's Disease -Self Rated, QOL-AD-PR: Quality of Life in Alzheimer's Disease -Proxy Rated, QoL-AD: Quality of Life in Alzheimer's Disease, $*$ : $p<0.05$.

In explaining the variance of QoL, it was revealed that MMSE, GDS and proxy-rated QoL explained $30 \%$ of the variance in self-rated QoL, whereas IADL and self-rated QoL explained $12 \%$ of the variance in proxyrated QoL. When examined in terms of QoL-AD, GDS explained 13\% of it (Table 3). 
Table 3

Regression models of factors predicting QOL-AD, self-rated QOL and proxy-rated QOL

\begin{tabular}{|c|c|c|c|c|c|}
\hline & & Beta coefficient & $\mathbf{R}^{2}$ & Adjusted $\mathrm{R}^{2}$ & $\mathrm{p}$ value \\
\hline \multirow[t]{3}{*}{ QOL-AD-SR } & MMSE & $-.274(p=.003)$ & \multirow[t]{3}{*}{.333} & \multirow[t]{3}{*}{.304} & \multirow[t]{3}{*}{$<.001 *$} \\
\hline & GDS & $-.681(p<.001)$ & & & \\
\hline & QOL-AD-PR & $.211(p=.009)$ & & & \\
\hline \multirow[t]{2}{*}{ QOL-AD-PR } & QOL-AD-SR & $.313(p=.029)$ & \multirow[t]{2}{*}{.141} & \multirow[t]{2}{*}{.117} & \multirow[t]{2}{*}{$.005^{*}$} \\
\hline & IADL & $.700(p=.019)$ & & & \\
\hline QOL-AD & GDS & $-.541(p=.001)$ & .146 & .134 & $.001 *$ \\
\hline
\end{tabular}

MMSE: Mini Mental State Examination, GDS: Geriatric Depression Scale, IADL: Instrumental Activities of Daily Living, QOL-AD-SR: Quality of Life in Alzheimer's Disease -Self Rated, QOL-AD-PR: Quality of Life in Alzheimer's Disease -Proxy Rated, QoL-AD: Quality of Life in Alzheimer's Disease, *: $p<0.05$.

\section{Discussion}

In this study, patients rated significantly higher QoL than the caregivers, which is in accordance with the literature.

Cognition, sleepiness and depression were associated with the self-rated QoL, while basic and instrumental activities of daily living were associated with the proxy rated QoL.

PwAD described a better quality of life as their cognitive level decreased. Poor quality of life was found to be associated with daytime sleepiness and depressive symptoms. In terms of caregivers, a more independent life means a better quality of life for the PwAD. If the total score of the quality-of-life scale were taken into account, it would be seen that only the patient's depressive symptoms negatively affected his quality of life and also there would be seen no relationship with other symptoms. For these reasons, both the reports of patients and proxies should be considered.

According to the results of our study, self- and proxy rated QoL are not related to age and disease duration. In previous studies, it is seen that the effect of age and duration of illness is a controversial issue on QoL of PwAD $(11,12,19,21,22)$. Similar to our study, Dewitte et al (12) showed there is no relationship between age and quality of life, but, unlike our results, Andrieu et al (11) and Hongisto et al (22) have shown that proxy rated QoL is affected by the disease duration. According to these 2 and 5year follow-up studies, the quality of life reported by the patient's caregiver has significantly decreased due to disease progression, functional and behavioral disorders. On the other hand, PwAD, did not change the quality of life during the disease process as they developed a mechanism to cope with the new 
difficulties they encountered. Decreasing patient awareness ensures that the quality of life is not affected by the disease duration (21).

Our results show that, caregivers assumed the patient's QoL with impaired cognition worsens, but this relationship was not statistically significant. When examined from the perspective of the patient, it is seen that the quality of life increases with cognitive impairments, supporting the literature (23-25). In Stites at al.'s study, PwAD who are aware of the diagnosis and the disease report more stress and depression, and poorer quality of life. Quality of life does not show a linear relationship with the disease. In a study comparing mild cognitive impairment $(\mathrm{MCl})$, mild $\mathrm{AD}$, and healthy individuals, $\mathrm{MCl}$ patients reported worse quality of life than AD and healthy individuals (25). According to this study of Stites et al., in the $\mathrm{MCl}$ grup awareness is high because their cognitive loss is lower, and therefore the perception of quality of life is poor. In our study, the negative correlation between quality of life and MMSE supports this result. As a health policy, early diagnosis is important, but when we consider our results, how to inform the patient with early diagnosis is also an important situation. Information should be given about psychological changes, in addition to the physical changes that may be seen in patients. Thus, in the earlier stages when there is awareness, there may be a milder situation.

Depression and sleepiness, that are consistent with previous research, were also correlated and contributed to lower self-rated QoL $(9,26-29)$. Depression, which is perceived and evaluated personally, is an important risk factor that also affects the quality of life, decreasing the brain's ability to cope with disease. As stated in previous studies, severe PwAD reject depressive symptoms, while mild to moderate patients show psychological distress. For these reasons, depression should be considered as a treatable condition from the early stages when awareness is higher and should be treated with the most appropriate approaches for the patient.

Similar to our study, Barbe et al, that measures the quality of life with OoL-AD (3 scores; patient, caregiver and overall), examined the associations of between the depression and the 13 items of the QoL-AD (30). In the both patient reports and caregiver reports, the presence of depression influenced the 3 items of QoL which consist of mood, ability to do things for fun and life as a whole. Instead of an extra depression evaluation, using these quality-of-life items may provide convenience. Studies are needed for this aim.

Another result of our study is that there is an association between the high level of daytime sleepiness and the worse quality of life. Sleep disorders and abnormal circadian rhythms are important problems which are seen in 25-60\% of PWAD $(9,29,31)$. According to Petrovsky et al's state of the art review, although sleep disorders are common, there is no study that summarizes how they affect QoL (29). It also causes stress for the caregiver because of leading different health-related problems in patients (depression, heart disease, decrease in functional capacity, etc). As can be seen from our results, patient's socialization is reduced due to excessive daytime sleepiness and accordingly their own quality of life perception decreases. Sleep, should be considered as a treatable factor affecting quality of life and take a part of PwAD's routine evaluation. 
Activities of daily living are one of the important issues investigated in the dementia group $(12,31-35)$. In our study, according to the statement of PWAD caregivers, better quality of life of patients is related to their independence in activities of daily living.

Subjective Cognitive Disorders (SCD), is an important stage in which cognitive complaints occur before $A D$ diagnosis. Since psychological conditions such as depression-anxiety also cause SCD, it is difficult to distinguish at this stage. Roehr et al (34) found that if SCD diagnosed patients have disabilities in IADL, they are likely to be diagnosed with $A D$ in the future. Due to the awareness of caregivers, IADL is an important point that should not be neglected during the disease process.

Executive functions and ADLs are more important by the caregiver (12). According to the results of the Barbe et al.'s study, being able to move and use the telephone creates self-esteem and provides a better perceived quality of life (31). Therefore, it is important to create a rehabilitation programme for an activity related to quality of life.

There are some limitations should be considered while interpreting this study's results. Non-inclusion of severe PWAD is one of the limitations of this study. The rate of PWADs staying in nursing homes has also increased due to the increase in disease's incidence and care needs. Therefore, another limitation of this study was the participation of only PWADs living in the community. Considering these limitations for future studies, it is recommended to plan more comprehensive studies.

\section{Conclusion}

The most important goal of Alzheimer's disease treatment is to provide a better quality of life. Because of having a caregiver whose priorities are different from the patient, while evaluating the QoL, both the evaluations of the PWAD and proxy should be applied. Needs and wishes of both the patient and his proxy should be taken into account when planning the treatment program.

\section{Abbreviations}

AD: Alzheimer's Disease; WHO: World Health Organization; QoL: Quality of Life; PwAD: People with Alzheimer's Disease; ADL: Activities of Daily Living; IADL: Instrumental ADL; MMSE: Mini Mental State Examination; QoL-AD: Quality of Life in Alzheimer's Disease; QoL-AD-SR: QoL-AD Self-Rating; QoL-ADPR: QoL-AD Proxy Rating; CDR: Clinical Dementia Rating, ESS:Epworth Sleepiness Scale; GDS: Geriatric Depression Scale.

\section{Declarations}

\section{Authors' contributions}

EF wrote the protocol, design study, organized the data collection process, analyzed the data and writing the manuscript. SÖ revise and approve the protocol, reviewing and editing the manuscript. HY contributed 
to the data collection, entering of data, writing and drafting the manuscript. RA contributed to the data collection, entering of data, writing and drafting the manuscript GY revise and approve the protocol, critically reviewed and approved the manuscript for publication. All authors read and approved the final manuscript.

\section{Availability of data and materials}

The datasets used and/or analyzed during the current study are available from the corresponding author on reasonable request by elvanfelekoglu@gmail.com

\section{Ethics approval and consent to participate}

The Project was ethically approved by the ethical committee of the Dokuz Eylul University, Noninvasive Research Ethics Board (Turkey) (2017/01-27).

\section{Funding}

This study was financially supported by researchers.

\section{Financial Disclosure}

The authors declared that this study has received no financial support

\section{Consent for publication}

Not Applicable.

\section{Competing interests}

The authors have declared that they have no conflicts of interest.

\section{References}

1. Schumann C, Alexopoulos P, Perneczky R. Determinants of self- and carer-rated quality of life and caregiver burden in Alzheimer disease. Int J Geriatr Psychiatry. 2019;34(10):1378-85. doi:10.1002/gps.5126.

2. Sampaio A, Marques-Aleixo I, Seabra A, Mota J, Marques E, Carvalho J. Physical fitness in institutionalized older adults with dementia: Association with cognition, functional capacity and quality of life. Aging Clin Exp Res. 2020;32(11):2329-38. doi:10.1007/s40520-019-01445-7.

3. Maresova P, Hruska J, Klimova B, Barakovic S, Krejcar O. Activities of Daily Living and Associated Costs in the Most Widespread Neurodegenerative Diseases: A Systematic Review. Clin Interv Aging. 2020;15:1841-62. doi:10.2147/CIA.S264688.

4. Junge S, Gellert P, O'Sullivan JL, Möller S, Voigt-Antons JN, Kuhlmey A, Nordheim J. Quality of life in people with dementia living in nursing homes: validation of an eight-item version of the QUALIDEM 
for intensive longitudinal assessment. Qual Life Res. 2020;29(6):1721-30. doi:10.1007/s11136-02002418-4.

5. O'Shea E, Hopper L, Marques M, Gonçalves-Pereira M, Woods B, Jelley H, Verhey F, Kerpershoek L, Wolfs C, de Vugt M, Stephan A, Bieber A, Meyer G, Wimo A, Michelet M, Selbaek G, Portolani E, Zanetti $\mathrm{O}$, Irving K, Actifcare Consortium. A comparison of self and proxy quality of life ratings for people with dementia and their carers: a European prospective cohort study. Aging Ment Health. 2020;24(1):162-70. doi:10.1080/13607863.2018.1517727.

6. Zucchella C, Bartolo M, Bernini S, Picascia M, Sinforiani E. Quality of life in Alzheimer disease: a comparison of patients' and caregivers' points of view. Alzheimer Dis Assoc Disord. 2015; 29(1):5054. doi: 10.1097/WAD.0000000000000050. PMID: 24936799.

7. who.int [Internet] World Health Organization; 2020 [cited 2020 January 06]. Available from: https://www.who.int/tools/whoqol.

8. Farina N, King D, Burgon C, Berwald S, Bustard E, Feeney Y, Habibi R, Comas-Herrera A, Knapp M, Banerjee $S, M O D E M$ group. Disease severity accounts for minimal variance of quality of life in people with dementia and their carers: analyses of cross-sectional data from the MODEM study. BMC Geriatr. 2020;20(1):232. doi:10.1186/s12877-020-01629-1.

9. Song D, Yu DS, Li PW, He G, Sun Q. Correlates of Health-Related Quality of Life Among Chinese Older Adults with Mild Cognitive Impairment. Clin Interv Aging. 2019;14:2205-12. doi:10.2147/CIA.S227767.

10. Stypa V, Haussermann P, Fleiner T, Neumann S. Validity and Reliability of the German Quality of LifeAlzheimer's Disease (QoL-AD) Self-Report Scale. J Alzheimers Dis. 2020;77(2):581-90. doi:10.3233/JAD-200400.

11. Andrieu S, Coley N, Rolland Y, Cantet C, Arnaud C, Guyonnet S, Nourhashemi F, Grand A, Vellas B. PLASA group. Assessing Alzheimer's disease patients' quality of life: Discrepancies between patient and caregiver perspectives. Alzheimers Dement. 2016;12(4):427-37. doi:10.1016/j.jalz.2015.09.003.

12. Dewitte L, Vandenbulcke $M$, Dezutter J. Cognitive functioning and quality of life: Diverging views of older adults with Alzheimer and professional care staff. Int J Geriatr Psychiatry. 2018;33(8):107481. doi:10.1002/gps.4895.

13. Folstein MF, Folstein SE, McHugh PR. "Mini-mental state". A practical method for grading the cognitive state of patients for the clinician. J Psychiatr Res. 1975 Nov;12(3):189-98. doi: 10.1016/0022-3956(75)90026-6.

14. Hughes CP, Berg L, Danziger WL, Coben LA, Martin RL. A new clinical scale for the staging of dementia. Br J Psychiatry. 1982 Jun;140:566-72. doi:10.1192/bjp.140.6.566.

15. Logsdon RG, Gibbons LE, McCurry SM, Teri L. Assessing quality of life in older adults with cognitive impairment. Psychosom Med. 2002 May-Jun;64(3):510-9. doi:10.1097/00006842-20020500000016.

16. Collin C, Wade DT, Davies S, Horne V. The Barthel ADL Index: a reliability study. Int Disabil Stud. 1988;10(2):61-3. doi:10.3109/09638288809164103. 
17. Lawton MP, Brody EM. Assessment of older people: self-maintaining and instrumental activities of daily living. Gerontologist. 1969 Autumn;9(3):179 - 86.

18. Alden D, Austin C, Sturgeon R. A correlation between the Geriatric Depression Scale long and short forms. J Gerontol. 1989 Jul;44(4):P124-5. doi:10.1093/geronj/44.4.p124.

19. Johns MW. A new method for measuring daytime sleepiness: The Epworth Sleepiness Scale. Sleep. 1991;14(6):540-5.

20. Akpınar Söylemez B, Küçükgüçlü Ö, Akyol MA, Işık AT. Quality of life and factors affecting it in patients with Alzheimer's disease: a cross-sectional study. Health Qual Life Outcomes. 2020;18(1):304. doi:10.1186/s12955-020-01554-2.

21. Dourado MCN, Santos RL, Fischer A, Mograbi DC. Modeling Quality of Life in Alzheimer Disease: The Impact of Cognitive, Functional, and Mood Variables in Self and Carers' Perceptions. J Geriatr Psychiatry Neurol. 2020:891988720944235. doi:10.1177/0891988720944235.

22. Hongisto K, Hallikainen I, Selander T, Törmälehto S, Väätäinen S, Martikainen J, Välimäki T, Hartikainen S, Suhonen J, Koivisto AM. Quality of Life in relation to neuropsychiatric symptoms in Alzheimer's disease: 5-year prospective ALSOVA cohort study. Int J Geriatr Psychiatry. 2018;33(1):47-57. doi:10.1002/gps.4666.

23. Thorsen K, Dourado MCN, Johannessen A. Awareness of dementia and coping to preserve quality of life: a five-year longitudinal narrative study. Int J Qual Stud Health Well-being. 2020;15(1):1798711. doi:10.1080/17482631.2020.1798711.

24. Stites SD, Karlawish J, Harkins K, Rubright JD, Wolk D. Awareness of Mild Cognitive Impairment and Mild Alzheimer's Disease Dementia Diagnoses Associated with Lower Self-Ratings of Quality of Life in Older Adults. J Gerontol B Psychol Sci Soc Sci. 2017;72(6):974-85. doi:10.1093/geronb/gbx100.

25. Stites SD, Harkins K, Rubright JD, Karlawish J. Relationships Between Cognitive Complaints and Quality of Life in Older Adults with Mild Cognitive Impairment, Mild Alzheimer Disease Dementia, and Normal Cognition. Alzheimer Dis Assoc Disord. 2018;32(4):276-83. doi:10.1097/WAD.0000000000000262.

26. Lima S, Sevilha S, Pereira MG. Quality of life in early-stage Alzheimer's disease: the moderator role of family variables and coping strategies from the patients' perspective. Psychogeriatrics. 2020;20(5):557-67. doi:10.1111/psyg.12544.

27. Millenaar J, Hvidsten L, de Vugt ME, Engedal K, Selbæk G, Wyller TB, Johannessen A, Haugen PK, Bakker C, van Vliet D, Koopmans RT, Verhey FR, Kersten H. Determinants of quality of life in young onset dementia - results from a European multicenter assessment. Aging Ment Health. 2017;21(1):24-30. doi:10.1080/13607863.2016.1232369.

28. Conde-Sala JL, Turró-Garriga O, Portellano-Ortiz C, Viñas-Diez V, Gascón-Bayarri J, Reñé-Ramírez R. Self-Perceived Quality of Life Among Patients with Alzheimer's Disease: Two Longitudinal Models of Analysis. J Alzheimers Dis. 2016;52(3):999-1012. doi:10.3233/JAD-160040.

29. Petrovsky DV, McPhillips MV, Li J, Brody A, Caffeé L, Hodgson NA. Sleep disruption and quality of life in persons with dementia: A state-of-the-art review. Geriatr Nurs. 2018;39(6):640-5. 
doi:10.1016/j.gerinurse.2018.04.014.

30. Barbe C, Jolly D, Morrone I, Wolak-Thierry A, Dramé M, Novella JL, Mahmoudi R. Factors associated with quality of life in patients with Alzheimer's disease. BMC Geriatr. 2018;18(1):159.

doi:10.1186/s12877-018-0855-7.

31. Barbe C, Morrone I, Wolak-Thierry A, Dramé M, Jolly D, Novella JL, Mahmoudi R. Impact of functional alterations on quality of life in patients with Alzheimer's disease. Aging Ment Health. 2017;21(5):571-6. doi:10.1080/13607863.2015.1132674.

32. Chan CS, Slaughter SE, Jones CA, Wagg AS. Greater Independence in Activities of Daily Living is Associated with Higher Health-Related Quality of Life Scores in Nursing Home Residents with Dementia. Healthcare (Basel). 2015;3(3):503-18. doi:10.3390/healthcare3030503.

33. Clemmensen FK, Hoffmann K, Siersma V, Sobol N, Beyer N, Andersen BB, Vogel A, Lolk A, Gottrup H, $\mathrm{H} \emptyset \mathrm{gh}$ P, Waldemar G, Hasselbalch SG, Frederiksen KS. The role of physical and cognitive function in performance of activities of daily living in patients with mild-to-moderate Alzheimer's disease - a cross-sectional study. BMC Geriatr. 2020;20(1):513. doi:10.1186/s12877-020-01926-9.

34. Manson A, Ciro C, Williams KN, Maliski SL. Identity and perceptions of quality of life in Alzheimer's disease. Appl Nurs Res. 2020;52:151225. doi:10.1016/j.apnr.2019.151225.

35. Roehr S, Riedel-Heller SG, Kaduszkiewicz H, Wagner M, Fuchs A, van der Leeden C, Wiese B, Werle J, Bickel H, König HH, Wolfsgruber S, Pentzek M, Weeg D, Mamone S, Weyerer S, Brettschneider C, Maier W, Scherer M, Jessen F, Luck T. Is function in instrumental activities of daily living a useful feature in predicting Alzheimer's disease dementia in subjective cognitive decline? Int J Geriatr Psychiatry. 2019;34(1):193-203. doi:10.1002/gps.5010.

\section{Supplementary Files}

This is a list of supplementary files associated with this preprint. Click to download.

- STROBEchecklistcrosssectional.docx 